\title{
Fusion of Fingerprint and Face by using DWT and SIFT
}

\author{
Shekhar Karanwal \\ PEC University Chandigarh \\ \#261, block C, Kurukshetra hostel, \\ PEC Chandigarh, India
}

\author{
Davendra Kumar \\ PEC University Chandigarh \\ \#170, block C, Kurukshetra hostel, \\ PEC Chandigarh, India
}

\author{
Rohit Maurya \\ PEC University Chandigarh \\ \#179, block C, Kurukshetra hostel, \\ PEC Chandigarh, India
}

\begin{abstract}
Image fusion is a process to combine information from multiple images of the same scene. The result of image fusion will be a new image which is more suitable for human and machine perception or further tasks of image processing such as image segmentation, feature extraction and object recognition. This paper explains DWT (Discrete Wavelet Transform) or we can say decomposition of two images, after decomposition, fusion of decomposition is carried out which produces a fused image and then SIFT features extracted from the fused image. After extracting SIFT features matching is performed. Experimental Results shows that proposed method produces $95 \%$ accuracy.
\end{abstract}

\section{Wavelet decomposition, SIFT, Feature Extraction and Image matching. \\ Our implementation is done in matlab most of the work is carried out in image. This paper describes how image is decomposed by wavelet decomposition, how fusion is performed, how features are extracted from image and how matching is performed.}

\section{Security and Reliability}

Wavelet based image fusion is basically used to provide more security and reliability of image as compared to other techniques of fusion. It decomposes an image into various resolutions which provide approximate and detail coefficients of image, which is then further processed for feature extraction and matching.

\section{Keywords}

Multimodal biometric fusion, DWT, SIFT, Image fusion, image matching.

\section{INTRODUCTION}

In biometric, human identity verification systems seek considerable improvement in reliability and accuracy. Several biometric authentication traits are offering 'up-to the-mark' and negotiable performance in respect of recognizing and identifying users. However, none of the biometrics is giving cent percent accuracy. Multibiometric systems [1] remove some of the drawbacks of the uni-modal biometric systems by acquiring multiple sources of information together in an augmented group, which has richer detail. Utilization of these biometric systems depends on more than one physiological or behavioral characteristic for enrollment and verification/ identification [2]. Multi-resolution approach in wavelet is well suited to manage the different image resolutions. Many research works have studied on multi-resolution representation of signals and have established that multi-resolution information for a number of image processing applications including the image fusion. Wavelet coefficients coming from different images can be appropriately combined to obtain new coefficients, so that the information in source images is collected appropriately. The discrete wavelet transform (DWT) allows the image decomposition in different kinds of coefficients preserving the image information. A wavelet-based image fusion method is therefore required to identify the most important information in the input images and to transfer it into the fused image.

A multisensor multimodal biometric system fuses information at low level or sensor level of processing is expected to produce more accurate results than the systems that integrate information at a later stages, namely, feature level, matching score level, because of the availability of more richer and relevant information.

In the first section introduction is carried out, second section explains wavelet decomposition, third section explains SIFT feature extractor, fourth section explains how matching is performed, in fifth section flowchart for fusion is shown, sixth section evaluates result, conclusion is drawn in section seven, section 8 persons are acknowledged and section 9 is of references

\section{WAVELET DECOMPOSITION FOR IMAGES}

This process basically takes two images of face and finger after taking images wavelet decomposition is performed on both images followed by a fusion of decomposition of two images which produces fused image of low resolution. It has the capability to provide good localization for both frequencies and space domains. The wavelet based image fusion would be applied to two dimensional multispectral face and fingerprint at each level. Consider two images

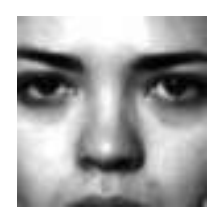

Figure 1.Face 


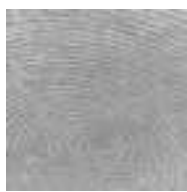

Figure 2.Fingerprint
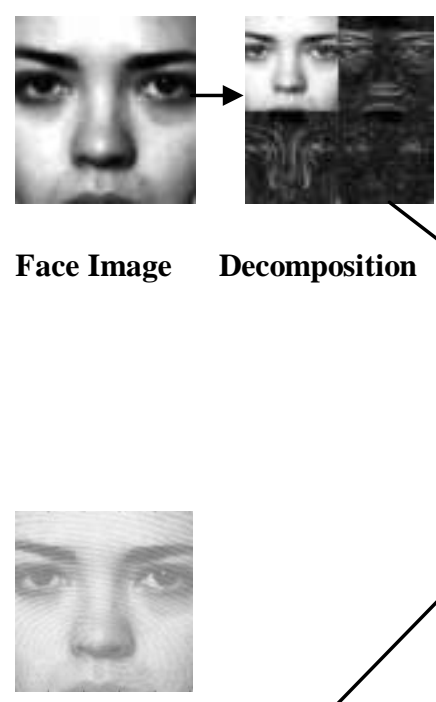

Decomposition

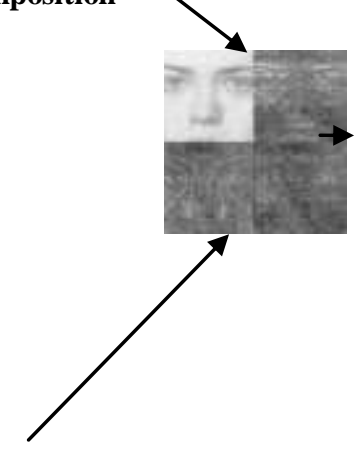

Fusion of Fused Image

Decomposition

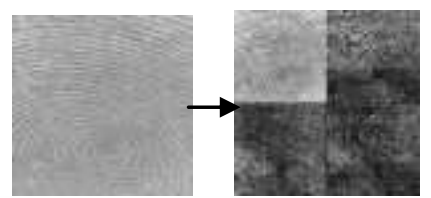

Finger Image Decomposition

Fig 3.Wavelet based face and Fingerprint images decomposition and Fusion of decompositions to a complete fused image

\section{SIFT (SCALE INVARIANT FEATURE TRANSFORM)}

The scale invariant feature transform, called SIFT [3] descriptor, has been proposed by and proved to be invariant to image rotation, scaling, translation, partly illumination changes. Following are the major stages of computation used to generate the set of image features

3.1. Scale space extrema detection: The first stage of computation searches over all scales and image locations. It is implemented efficiently by using a difference-of-Gaussian function to identify potential interest points that are invariant to scale and orientation.
3.2. Keypoint localization: At each candidate location, a detailed model is fit to determine location and scale. Keypoints are selected based on measures of their stability.

3.3. Orientation assignment: One or more orientations are assigned to each keypoint location based on local image gradient directions. All future operations are performed on image data that has been transformed relative to the assigned orientation, scale, and location for each feature, thereby providing invariance to these transformations.

3.4. Keypoint descriptor: The local image gradients are measured at the selected scale in the region around each keypoint. These are transformed into a representation that allows for significant levels of local shape distortion and change in illumination.

This approach has been named the Scale Invariant Feature Transform (SIFT), as it transforms image data into scaleinvariant coordinates relative to local features. An important aspect of this approach is that it generates large numbers of features that densely cover the image over the full range of scales and locations.

In this paper only the keypoint descriptor information is taken from image but before this fused image is normalized by histogram equalization, after invariant SIFT features are extracted from the fused image.

For image matching and recognition, SIFT features are first extracted from a set of reference images and stored in a database. A new image is matched by individually comparing each feature from the new image to this previous database and finding candidate matching features based on their feature vectors.

The keypoint descriptors are highly distinctive, which allows a single feature to find its correct match with good probability in a large database of features. However, in a cluttered image, many features from the background will not have any correct match in the database, giving rise to many false matches in addition to the correct ones. The correct matches can be filtered from the full set of matches by identifying subsets of Keypoints that agree on the object and its location, scale, and orientation in the new image. SIFT features extracted on the fused image is shown below.

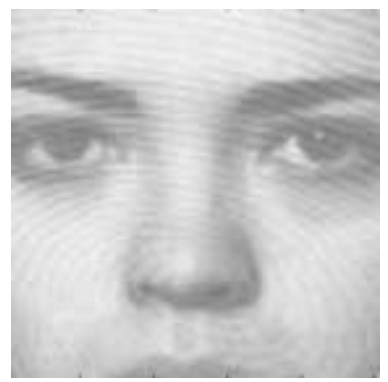

Figure 4.Fused Image 


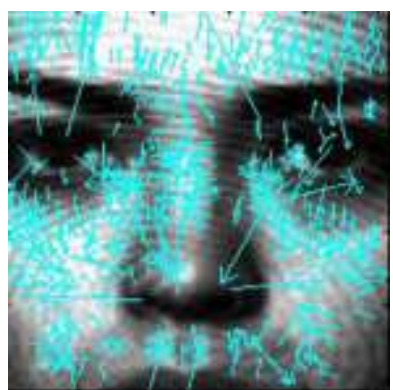

Figure 5.SIFT Features extracted from the fused Image

\section{STEPS OF MATCHING USING UNIT VECTORS}

1. Match (image1, image2). This function reads two images, finds their SIFT [4] [5] [6] features, and displays lines connecting the matched Keypoints. A match is accepted only if its distance is less than dist Ratio times the distance to the second closest match. It returns the number of matches displayed.

2. Find SIFT (Scale Invariant Fourier Transform) Keypoints for each image. For finding the SIFT Keypoints specify what are its locations and descriptors.

3. It is easier to compute dot products between unit vectors rather than Euclidean distances. Note that the ratio of angles acos of dot products of unit vectors is a close approximation to the ratio of Euclidean distances for small angles.

4. Assume some distance ratio for example suppose distance ratio=. 4 it means that it only keep matches in which the ratio of vector angles from the nearest to second nearest neighbor is less than distance Ratio.

5. Now for each descriptor in the first image, select its match to second image.

6. Compute matrix transpose, Computes vector of dot products, Take inverse cosine and sort results. Check if nearest neighbor has angle less than dist Ratio times 2 nd.

7. Then create a new image showing the two images side by side.

8. Lastly a figure is shown with lines joining the accepted matches.

Now apply these steps in our previous image from which SIFT features are extracted.

Now suppose that these extracted feature points are stored in the database, if a person comes he repeat same steps which are described above. After applying these steps to our image, matching image looks like this.

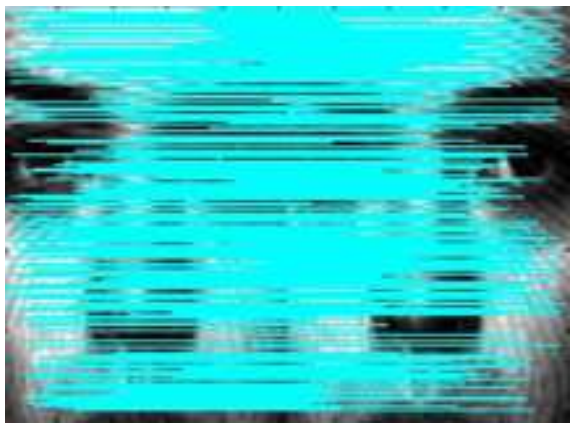

Figure 6.Matching

These are the all matching points of a person indicating that a person is authentic.

So same procedure repeats for every person, for every person its Keypoints are extracted after extracting Keypoints it is stored in the database and for matching, same procedure follows.

\section{FLOWCHART FOR FUSION OF FINGERPRINT AND FACE}

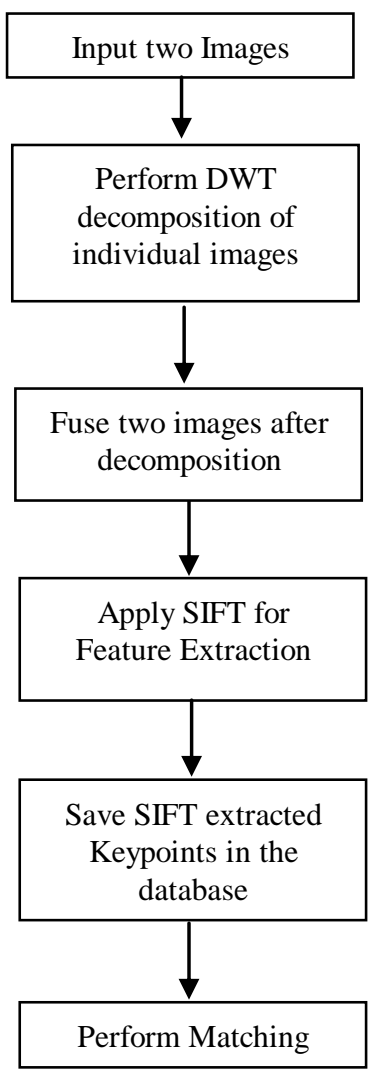




\section{EVALUATING RESULTS}

The experiment of the proposed method is carried out on a database of 50 persons. The face and fingerprint database are collected for individuals by taking the image of $256 \times 256$ and resolution is set to 72 dpi. For the sake of the experiment cropped face has been taken which covers face only and for the fingerprint cropped fingerprint has been taken which covers ridges and lines. Decomposition is done by DWT, after obtaining the fused images of face and fingerprint, fused image is preprocessed by histogram equalization for normalization. Finally Matching is performed between a pair of fused images by unit vectors. The matching is accomplished for proposed method and the result shows that it produces $95 \%$ accuracy.

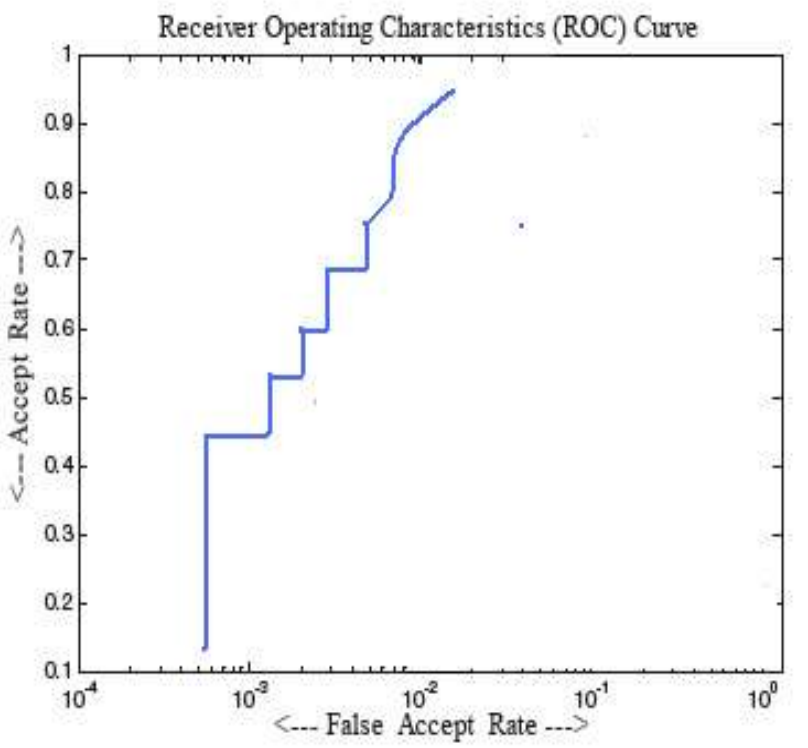

Figure 7.ROC Curve for False Acceptance Rate Vs Accept Rate are shown

The ROC curves shown in above figure illustrate the trade-off between accept rate and false accept rate. Further it shows that the increase in accept rate is accompanied by decrease in false accept rate. Blue line indicates biometric fusion.

\section{CONCLUSION}

An efficient method for fusion of face and fingerprint is proposed. Proposed method first decompose images and then fused image is produced, which is then further pre-processed by histeq, then SIFT features are extracted from fused image and finally matching is performed. An experimental result shows that proposed method produces $95 \%$ accuracy.

\section{ACKNOWLDEGEMENTS}

I would like to thank all of my friends who supported me for completing this template. Without their help it is very difficult to complete this template. I would also like to thanks my college faculties who provide me data for my work.

\section{REFERENCES}

[1] A.K.Jain and A. Ross, "Multibiometric systems", Communications of the ACM, vol. 47, no.1, pp. $34-40$, 2004.

[2] Dakshina Ranjan Kisku, Jamuna Kanta Sing, MassimoTistarelli, Phalguni Gupta," Multisensor Biometric Evidence Fusion for Person Authentication using Wavelet Decomposition and Monotonic-Decreasing Graph", 2009 Seventh International Conference on Advances in Pattern Recognition, 2009 IEEE.

[3] D. G. Lowe, "Distinctive image features from scale invariant Keypoints", International Journal of Computer Vision, vol. 60, no. 2, 2004.

[4] D. G. Lowe, "Object recognition from local scale invariant features," International Conference on Computer Vision, Corfu, Greece, pp.1150 - 1157, September 1999.

[5] U. Park, S. Pankanti and A. K. Jain, "Fingerprint Verification Using SIFT Features", Proceedings of SPIE Defence and Security Symposium, Orlando, Florida, 2008.

[6] M. Bicego, A. Lagorio, E. Grosso and M. Tistarelli, "On the use of SIFT features for face authentication", Proc. of Int Workshop on Biometrics, in association with CVPR 2006. 